\title{
The Relationship Between the Admission Blood Glucose Level and 90-Day Mortality in Non-Diabetic Patients with Coronavirus Disease-2019
}

\author{
(1) Emre Sedar Saygılı, (1) Ersen Karakılıç \\ Çanakkale Onsekiz Mart University Faculty of Medicine, Department of Internal Medicine, Division of Endocrinology and Metabolism, Çanakkale, \\ Turkey
}

\begin{abstract}
Introduction: The admission blood glucose (ABG) level is associated with increased mortality in non-diabetics patients with Coronavirus disease-2019 (COVID-19) in short-term follow-up studies. However, post-discharge mortality has also increased in COVID-19. Thus, this study aimed to examine the relationship between ABG and 90-day mortality including the post-discharge period.

Methods: Non-diabetic patients who are hospitalized due to COVID-19 in 2020 were evaluated. Patients were divided into groups according to the ABG level. Groups 1, 2, and 3 have ABG level of $<100 \mathrm{mg} / \mathrm{dL}, 100-139 \mathrm{mg} / \mathrm{dL}$, and 140-199 mg/dL, respectively. Intensive care unit admission, in-hospital mortality, and 30- and 90-day mortality rates were evaluated as outcomes. COX regression analyzes were used to assess mortality risk factors.

Results: A total of 1207 non-diabetic patients, of whom $49.2 \%$ were females, with a mean age of $65.2 \pm 13.4$ years, were included in the study. The patients were followed up for a median of 153 (inter quartile range: 107.5-251, maximum: 369) days. The in-hospital and 30-day mortality of group 2 was higher than group 1 in the univariate analysis but without statistical significance in multivariate analysis. Group 3 had worse outcomes than group 1 in both univariate and multivariate analysis at all end points. Group 3 had 2.533 adjusted hazard ratios (95\% confidence interval: 1.628-3.941, $\mathrm{p}<0.001)$ 90-day mortality compared with group 1.

Conclusion: Non-diabetic patients with COVID-19 with an admission glucose level of $\geq 140 \mathrm{mg} / \mathrm{dl}$ had 2.5 -fold increased all-cause mortality at 90 days. Therefore, being more careful in treating and following non-diabetic patients with COVID-19, especially those with hyperglycemia at admission, was recommended.
\end{abstract}

Keywords: Glucose, SARS-CoV-2, COVID-19, hyperglycemia, hospitalization, intensive care units

\section{Introduction}

Hyperglycemia in hospitalized patients is an independent risk factor that is strongly associated with adverse outcomes. Hyperglycemia disrupts neutrophil functions and movements, causing adverse effects on immunity. Additionally, it causes microvascular complications with endothelial dysfunction by increasing inflammatory responses and oxidative stress $(1,2)$.

Many associated factors with mortality have been identified in patients hospitalized due to Coronavirus disease-2019 (COVID-19). Mortality rates increased nearly 2 -fold in patients with a history of diabetes (36). Additionally, newly detected hyperglycemia in-hospital admission, even without a known history of diabetes, has shown association with increased mortality $(7,8)$, as well as increased intensive care unit (ICU) admissions and prolonged hospital stays. Several studies have shown that patients with hyperglycemia without a history of diabetes have an even higher risk of mortality than those with known diabetes (9).
Studies that examined the newly developed hyperglycemia at hospital admission due to COVID-19 mainly examined in-hospital and 30-day mortality rates. These studies did not evaluate the long-term mortality $(7,10)$. However, the mortality also increased in the post-discharge period in patients with COVID-19, and this rate has been reported to be approximately $10 \%$ (11-13). This study aimed to evaluate the relationship between at least 90 days mortality and admission blood glucose (ABG) level, including the post-discharge period.

\section{Methods}

A total of 1207 hospitalized patients with COVID-19 between March 2020 and December 2020 in Çanakkale Onsekiz Mart University Hospital were retrospectively analyzed. COVID-19 diagnoses were made either by polymerase chain reaction positivity or radiological findings. Patients with compatible diabetes diagnoses from the previously given International Classification of Diseases (ICD) codes, hemoglobin A1c (HbA1c) of $\geq 6.5 \%$ in 
their system records, using diabetes medication before admission, under 18 years of age, pregnant, and ABG of $>200 \mathrm{mg} / \mathrm{dL}$ were excluded from the study. Patients with newly diagnosed diabetes were also excluded when the $\mathrm{HbA} 1 \mathrm{c}$ is $\geq 6.5 \%$ during hospitalization. Patients were divided into groups according to the ABG level. Groups 1, 2, and 3 have ABG level of $<100 \mathrm{mg} / \mathrm{dL}$ (5.6 mmol/L), 100-139 mg/dL (5.6-7.7 mmol/L), and 140-199 mg/dL (7.8-11.1 mmol/L), respectively. The study was approved by the Ethics Committee of Çanakkale Onsekiz Mart University Faculty of Medicine (approval number: 2021-07, date: 20.10.2021).

Patients' mortality status was inquired via the Turkish Central Death Registry in March 2021. ICU admission, in-hospital mortality, and 30and 90-day mortality rates were evaluated as outcomes.

Acute renal failure was diagnosed by Kidney Disease: Improving Global Outcomes criteria (14). The Chronic Kidney Disease Epidemiology Collaboration equation was used to calculate the estimated glomerular filtration rate (eGFR) (15).

\section{Statistical Analysis}

Continuous variables with normal distribution were presented as mean \pm standard deviation, whereas non-normally distributed variables as the median and interquartile range [(IQR): the difference between the $25^{\text {th }}$ and $75^{\text {th }}$ percentiles]. Numbers and percentages were used to express categorical variables. Normal distribution was evaluated with the Kolmogorov-Smirnov test. Analysis of variance was used to compare the three groups for data with normal distribution and the Kruskal-Wallis test used for those with non-normal distribution. Homogeneity of variances was evaluated with Levene's test. Pairwise post-hoc comparisons were made with the Tukey test if the variances were homogeneous and with the Tamhane T2 test if not if a significant difference was found between the three groups. In the variables, in which the groups were evaluated with the Kruskal-Wallis test, post-hoc analyses in pairs were evaluated using the Mann-Whitney $\mathrm{U}$ test by Bonferroni correction. Pearson's chi-square was used to test the distribution among categorical variables. Wherein, paired post-hocs were evaluated with the Z-test, with Bonferroni correction.

COX regression analyses were used to assess the risk factors for COVID19-associated mortality. A single variable was used to calculate the unadjusted hazard ratios (HR). Gender, age, alanine transaminase (ALT), eGFR, co-morbidities as chronic lung disease, coronary artery disease, cerebrovascular disease, hypertension, and hyperlipidemia were used in the adjusted HR (aHR) analysis. In COX regression analyses, group 1 was used as the reference group to evaluate the outcomes of groups 2 and 3 .

The Statistical Package for the Social Sciences version 19 for Windows was used for statistical analysis (IBM, Armonk, NY, USA). A statistically significant $p$-value of 0.05 was used.

\section{Results}

A total of 1207 patients with a mean age of $65.2 \pm 13.4$ years, of whom 49.2\% (594) were females, were included in the study. In all study groups, the in-hospital mortality rate was $11.1 \%$ (134). The 30 - and 90-day mortality rates were $12.7 \%$ (153) and 16.7\% (201), respectively.
Groups 1, 2, and 3 consisted of 285 (23.6\%), 706 (58.5\%), and 216 (17.9\%) patients, respectively.

From groups 1 to 3, ICU Admission, in-hospital mortality, 30-day mortality, and 90-day mortality rates increased as blood sugar increased. In the post-hoc analysis, these differences were determined to be caused mainly by group 3. From groups 1 to 3 , a decreased eGFR and a prolonged hospitalization were observed. Group 3 had higher ALT levels, hyperlipidemia history, and acute renal failure rates than other groups. Group 1 was younger and had a lower coronary artery disease rate. The general characteristics and comparisons of the groups are given in Table 1.

In-hospital mortality and 30-day mortality rates, which were significant for mortality in the univariate analysis for group 2 (ABG: 100-139 mg/dL), lost statistical significance in the multivariate analysis adjusted for multiple variables (gender, age, ALT, eGFR, and co-morbidities). In group 3, univariate analysis of 90-day mortality HR was 3.45 [95\% confidence interval (CI): 2.231-5.335, $\mathrm{p}<0.001]$ and in the multivariate analysis of 90-day mortality aHR was 2,533 (95\% Cl: 1.628-3.941, $\mathrm{p}<0.001)$. In group 3 (ABG: 140-199 $\mathrm{mg} / \mathrm{dL}$ ), statistically, significant mortality increases were observed with all univariate and multivariate analysis outcomes. Univariate and multivariate HR and forest plot graphs of groups 2 and 3 according to reference group 1 are given in Figure 1 and 2, respectively.

Patients were followed up for a median of 153 (IQR: 107.5-251, minimum: 1, maximum: 369) days. The Kaplan-Meier one minus survival graph of mortality between the groups during the follow-up periods is given in Figure 3 (log-rank $p<0.001)$. The all-cause mortality rate was the highest in group 3 during the follow-up period.

\section{Discussion}

We found that ICU admission, in-hospital, 30-day, and 90-day all-cause mortality was higher in non-diabetic patients with ABG of 140-199 mg/dL, even after correction for other factors in our study, which had a maximum follow-up period of close to 1 year. Our study is among the first reports that investigated the effects of $A B G$ on long-term mortality in non-diabetic patients with COVID-19 (16).

Our findings are consistent with those of other studies with a shortterm follow-up that found increased mortality due to hyperglycemia in non-diabetic patients with COVID-19 $(7,10,17)$. Unlike these studies, our study showed the effect of ABG level on long-term mortality. Mortality rates for the post-discharge period in patients with COVID-19 have been reported as approximately 10\% (11-13). Therefore, long-term follow-up studies of patients are also important.

Diabetes mellitus, one of the most common co-morbidities in patients with COVID-19, has been reported from $7 \%$ to $30 \%$ in studies. People with diabetes with COVID-19 have higher hospital admission rates, severe pneumonia, and higher mortality than non-diabetics (18). Hyperglycemia that occurs during acute illness, called stress hyperglycemia, is also associated with adverse outcomes (19).

Various studies before the COVID-19 era have shown that high ABG is associated with increased mortality and morbidity in patients 
Table 1. General characteristics and comparisons of the admission blood glucose groups

\begin{tabular}{|c|c|c|c|c|c|}
\hline & $\begin{array}{l}\text { Group } 1 \text { ABG }<100 \\
\mathrm{mg} / \mathrm{dL}\end{array}$ & $\begin{array}{l}\text { Group } 2 \text { ABG } 100- \\
139 \mathrm{mg} / \mathrm{dL}\end{array}$ & $\begin{array}{l}\text { Group } 3 \text { ABG 140- } \\
199 \mathrm{mg} / \mathrm{dL}\end{array}$ & Total & p \\
\hline Number of patients & 285 & 706 & 216 & 1207 & - \\
\hline Age (years) & $61.0 \pm 14.7^{* * * *}$ & $66.1 \pm 13.1$ & $67.8 \pm 11.9$ & $65.2 \pm 13.5$ & $<0.001$ \\
\hline Female (\%) & $142(49.8)$ & $343(48.6)$ & $109(50.5)$ & $594(49.2)$ & 0.865 \\
\hline Glucose (mg/dL) & $91.1 \pm 6.3$ & $116.5 \pm 10.4$ & $160.4 \pm 16.3$ & $118.4 \pm 24.8$ & $<0.001$ \\
\hline Number of hospitalization days [median (IQR)] & $5(4-10)^{*, * *}$ & $6(5-10)$ & $7(4-13)$ & $6(4-10)$ & 0.01 \\
\hline Follow-up days count [median (IQR)] & $204(130-260)^{*}$ & $150(109-256)^{* * * *}$ & $125.5(32.5-228)^{* *}$ & $153(107.5-251)$ & 0.01 \\
\hline In-hospital mortality (n, \%) & $16(5.6)$ & $73(10.3)$ & $45(20.8)^{* * *}$ & $134(11.1)$ & $<0.0001$ \\
\hline COPD-asthma (n, \%) & $43(15.1)$ & $111(15.7)$ & $36(16.7)$ & $190(15.7)$ & 0.891 \\
\hline Hypertension (n, \%) & $104(36.5)$ & $298(42.2)$ & $99(45.8)$ & $501(41.5)$ & 0.09 \\
\hline Coronary artery disease (n, \%) & $37(13)^{*, * * *}$ & $144(20.4)$ & $59(27.3)$ & $240(19.9)$ & $<0.0001$ \\
\hline Hyperlipidemia (n, \%) & $28(9.8)$ & $83(11.8)$ & $41(19)^{* * * * * *}$ & $152(12.6)$ & 0.005 \\
\hline Acute renal failure (n, \%) & $41(14.4)$ & $11(16)$ & $49(22.7)^{* * *}$ & $203(16.8)$ & 0.03 \\
\hline Chronic renal failure (n, \%) & $8(2.8)$ & $23(3.3)$ & $1(5.6)$ & $43(3.6)$ & 0.2 \\
\hline Cerebrovascular disease (n, \%) & $15(5.3)$ & $50(7.1)$ & $16(7.4)$ & $8(6.7)$ & 0.52 \\
\hline ACE/ARB usage $(n, \%)$ & $74(26)$ & $229(32.4)$ & $71(32.9)$ & $374(31)$ & 0.11 \\
\hline
\end{tabular}

hospitalized for trauma, surgery, infectious diseases (sepsis and pneumonia), cardiovascular diseases, and cerebrovascular diseases (2023). Even without a history of diabetes, the risk of mortality is higher in cases with the hyperglycemic course during hospitalization. Some studies also revealed that newly developed hyperglycemia without a history of diabetes during hospitalization was related to higher mortality than known patients with diabetes (9). Angiotensin-Converting Enzyme II (ACE-2) is one of the receptors that allow the entry of the severe acute respiratory syndrome-coronavirus-2 (SARS-COV-2) into the cell. Acute hyperglycemia increases ACE-2 expression, whereas chronic hyperglycemia decreases ACE-2 expression (24). It may explain why newly developed hyperglycemia has a worse prognosis than those with known diabetes. Additionally, patients with hyperglycemia with known diabetes are likely to be promptly treated, and the fact that hyperglycemia is a marker of critical diseases that may increase mortality may also explain this condition (25).

The relationship between long-term mortality with ABG was also investigated in cardiovascular diseases before the COVID-19 era $(21,26,27)$. Similar to our findings, ABG was associated with increased long-term mortality.

The causes of hyperglycemia during hospitalization can be listed as treatments that contain dextrose, glucocorticoid, increased anti-insulin hormones, and increased inflammatory response $(28,29)$. Our study was conducted according to the first ABG level groups, thus the effect of secondary hyperglycemia due to glucocorticoid treatment on the analysis was prevented (17).

Hyperglycemia induces an exaggerated inflammatory response that results in harmful microvascular complications (30). Additionally, hyperglycemia can affect multiple biochemical pathways that can facilitate many steps of SARS-CoV-2 infection. Hyperglycemia may trigger ACE-2 receptor expression, which may increase the susceptibility to COVID-19 infection and the risk of severe disease and multi-organ failure (31). The association of hyperglycemia with increased mortality is multifactorial (1).

Some differences were determined in the definition of hyperglycemia in studies that examine the effect of hyperglycemia on mortality in nondiabetics. Some studies used a definition of hyperglycemia above 140 $\mathrm{mg} / \mathrm{dL}$, thus patients with a level above $200 \mathrm{mg} / \mathrm{dL}$ were not excluded in some studies. Our study excluded the ABG of $>200 \mathrm{mg} / \mathrm{dL}$ to eliminate the overlap with the diagnosis of diabetes.

Age, male gender, multiple pre-existing co-morbidities, and renal/ hepatic dysfunction are critical factors that predict COVID-19 mortality (32). Therefore, a factor should be corrected in terms of the main influencing factors to correctly evaluate the relationship with mortality. Univariate analysis of ABG of 100-140 mg/dL was significant for mortality, but without significance, after the multivariate analysis was adjusted for 


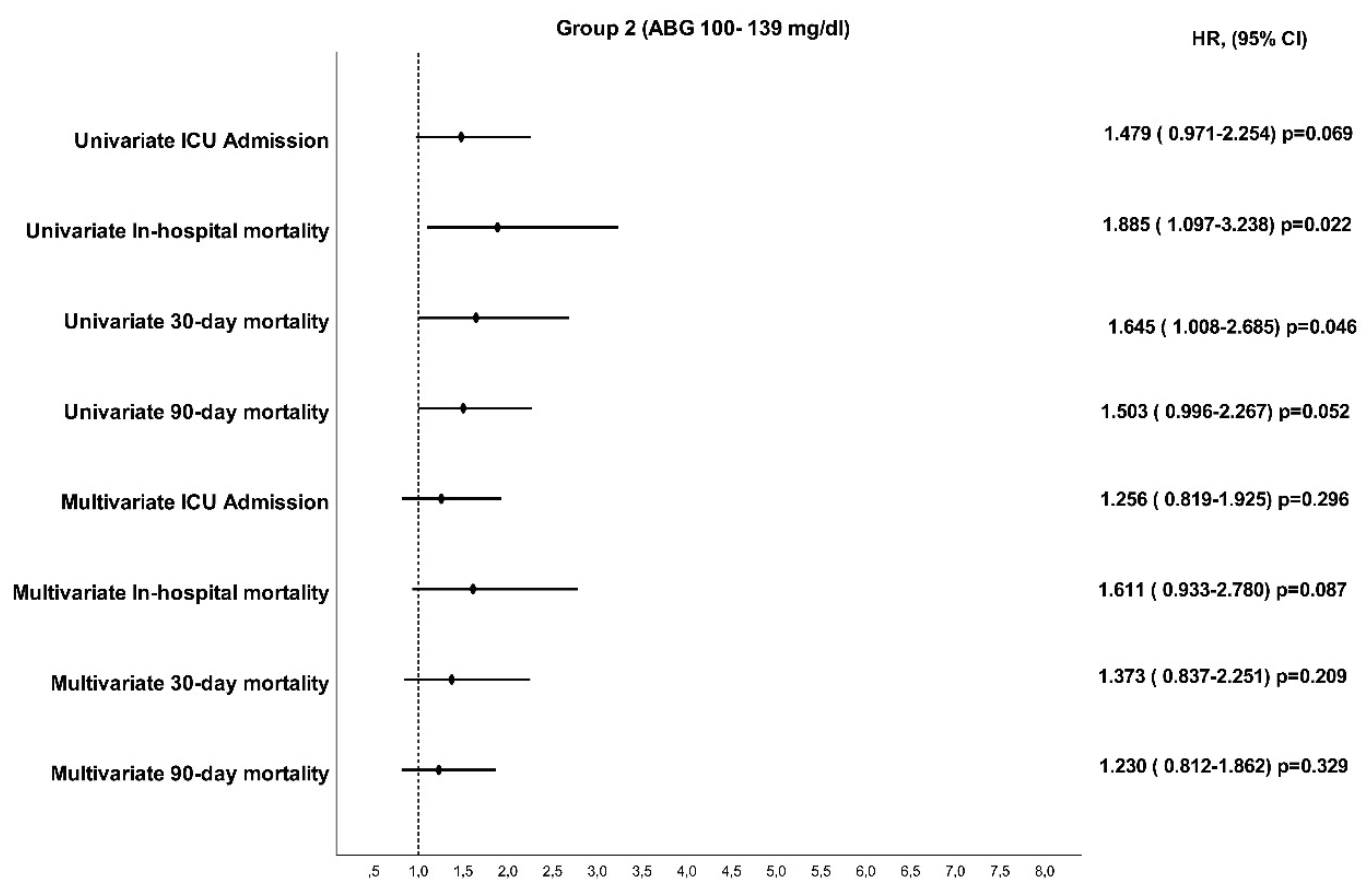

Figure 1. Univariate and multivariate hazard ratios and forest plot graph of group 2 according to the reference group 1 ABG: Admission blood glucose, HR: hazard ratios, Cl: confidence interval, ICU: intensive care unit

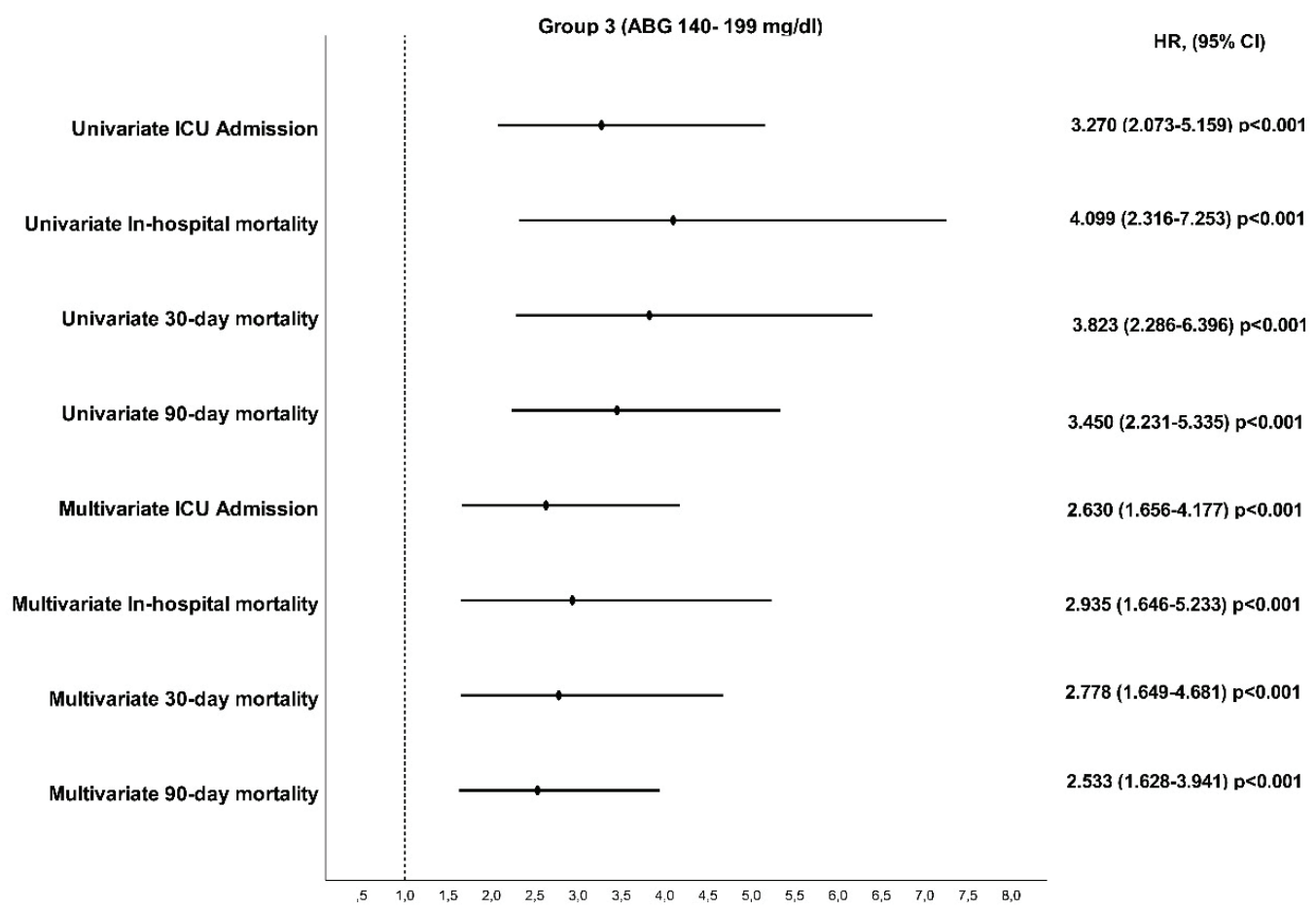

Figure 2. Univariate and multivariate hazard ratios and forest plot graph of group 3 according to the reference group 1

ABG: Admission blood glucose, HR: hazard ratios, Cl: confidence interval, ICU: intensive care unit

covariates. The effect of mildly elevated blood sugar (100-140 mg/dL) on mortality can be ignored. The guidelines defined hyperglycemia as any glucose level above $140 \mathrm{mg} / \mathrm{dL}$ in hospitalized patients (33,34). Other studies revealed that blood sugar influences mortality when it is $>140$ $\mathrm{mg} / \mathrm{dL}(10,21)$.

\section{Study Limitations}

Our study has several limitations. There may be undetected confounding factors because it is a retrospective study of data during an outbreak. Patients without diabetes-related ICD codes, medications history, and reports were selected; however, the fact that the HbA1c measurement 


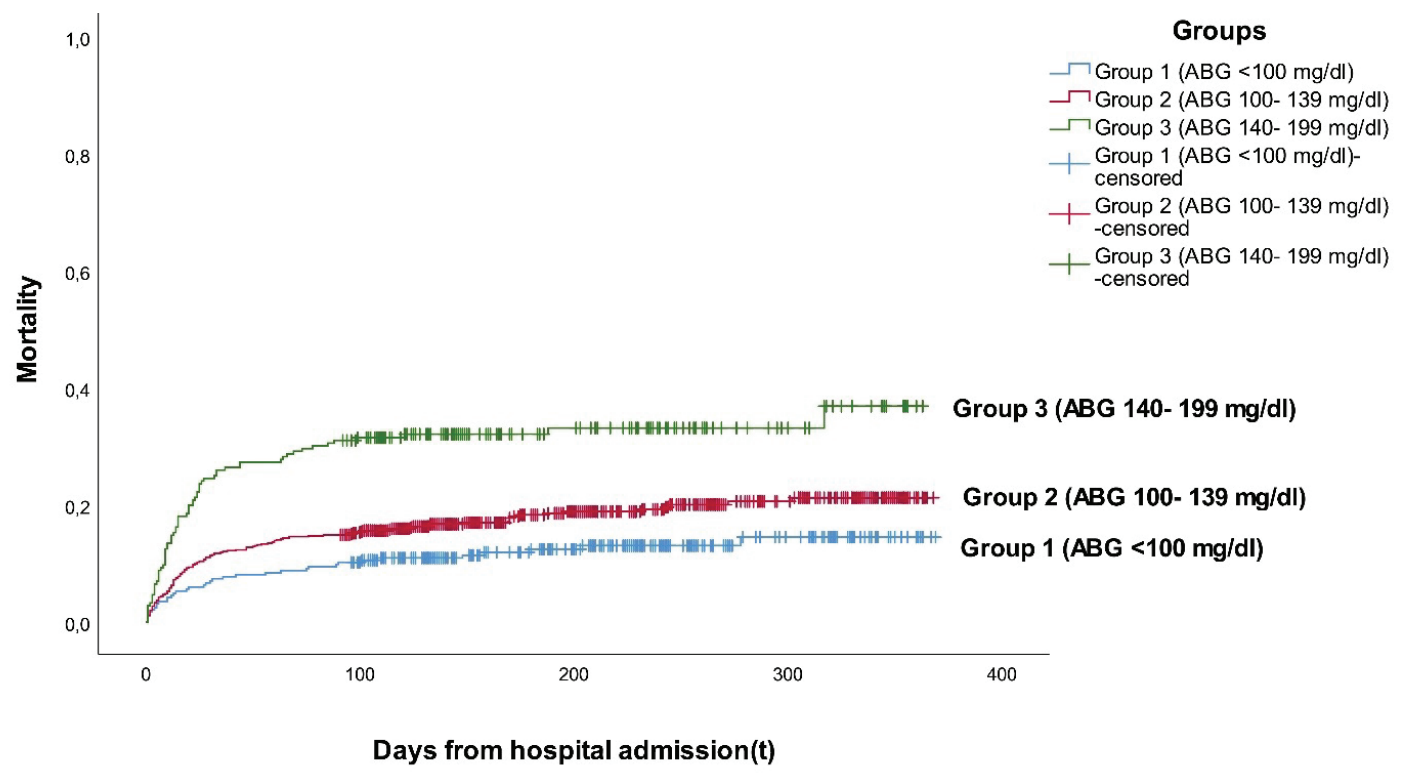

Figure 3. The Kaplan-Meier one minus survival graph of groups (log-rank $p<0.001)$

ABG: Admission blood glucose

was scarce among the patients is a limiting factor in excluding the history of diabetes. The ABG value taken in the fasting state or randomly could not be determined. However, our study examined the effect of ABG level on mortality rather than diagnosing new diabetes. All-cause mortality was evaluated in the analysis, thus data on exact causes of death were insufficient. The strengths of our study are the large, real-life cohort of hospitalized patients with COVID-19 and the long-term follow-up. Whether hyperglycemia treatment improves outcomes in those with high ABG levels requires a different study design. Following the guidelines, when the hospitalized patient's blood sugar levels reached $>180 \mathrm{mg} / \mathrm{dL}$, insulin was administered to lower the blood sugar level (34).

\section{Conclusion}

Non-diabetic COVID-19 patients with an admission glucose level of $\geq 140 \mathrm{mg} / \mathrm{dL}$ had a 2.5 -fold increased all-cause mortality at 90 days. Being more careful in treating and following a non-diabetic patient with COVID-19, especially those with elevated serum glucose at admission, is recommended.

Acknowledgments: We acknowledge and thank all of the doctors, nurses and other medical personnel at our hospital who provided care to patients during the COVID-19 pandemic. Especially, we would like to thank Dr. Erdal Mert for his support.

Ethics Committee Approval: The study was approved by the Ethics Committee of Çanakkale Onsekiz Mart University Faculty of Medicine (approval number: 2021-07, date: 20.10.2021).

Informed Consent: Retrospective study.

Peer-review: Externally peer-reviewed.

Authorship Contributions: Concept - E.S.S.; Design - E.S.S.; Data Collection or Processing - E.S.S., E.K.; Analysis or Interpretation - E.S.S., E.K.; Literature Search - E.S.S., E.K.; Writing - E.S.S., E.K.
Conflict of Interest: No conflict of interest was declared by the authors.

Financial Disclosure: The authors declared that this study received no financial support.

\section{References}

1. Prieto-Sanchez LM. Hyperglycemia in-hospital management. Therapeutic Advances in Endocrinology and Metabolism 2011; 2: 3-7.

2. Forsblom E, Ruotsalainen E, Järvinen A. Prognostic impact of hyperglycemia at onset of methicillin-sensitive Staphylococcus aureus bacteraemia. Eur J Clin Microbiol Infect Dis 2017; 36: 1405-13.

3. Sulu C, Kadıoğlu P. Dealing with COVID-19: Through Endocrinologist's Eyes. Turk J Endocrinol Metab 2020; 24: 335-42.

4. Apicella M, Campopiano MC, Mantuano M, Mazoni L, Coppelli A, Del Prato S. COVID-19 in people with diabetes: understanding the reasons for worse outcomes. Lancet Diabetes Endocrinol 2020; 8: 782-92.

5. Guo W, Li M, Dong Y, Zhou H, Zhang Z, Tian C, et al. Diabetes is a risk factor for the progression and prognosis of COVID -19. Diabetes Metab Res Rev 2020; 36: e3319.

6. Barron E, Bakhai C, Kar P, Weaver A, Bradley D, Ismail H, et al. Associations of type 1 and type 2 diabetes with COVID-19-related mortality in England: a whole-population study. Lancet Diabetes Endocrinol 2020; 8: 813-22.

7. Wang S, Ma P, Zhang S, Song S, Wang Z, Ma Y, et al. Fasting blood glucose at admission is an independent predictor for 28-day mortality in patients with COVID-19 without previous diagnosis of diabetes: a multi-centre retrospective study. Diabetologia 2020; 63: 2102-11. 
8. Lazarus G, Audrey J, Wangsaputra VK, Tamara A, Tahapary DL. High admission blood glucose independently predicts poor prognosis in COVID-19 patients: A systematic review and dose-response metaanalysis. Diabetes Res Clin Pract 2021; 171: 108561.

9. Pratiwi C, Zulkifly S, Dahlan TF, Hafidzati A, Oktavia N, Mokoagow MI, et al. Hospital related hyperglycemia as a predictor of mortality in non-diabetes patients: A systematic review. Diabetes Metab Syndr 2021; 15: 102309.

10. Haymana C, Demirci I, Tasci I, Cakal E, Salman S, Ertugrul D, et al. Clinical outcomes of non-diabetic COVID-19 patients with different blood glucose levels: a nationwide Turkish study (TurCoGlycemia). Endocrine 2021; 73: 261-9.

11. Donnelly JP, Wang XQ, Iwashyna TJ, Prescott HC. Readmission and Death after Initial Hospital Discharge among Patients with COVID-19 in a Large Multihospital System. JAMA 2021; 325: 304-6.

12. Ayoubkhani D, Khunti K, Nafilyan V, Maddox T, Humberstone B, Diamond I, et al. Post-covid syndrome in individuals admitted to hospital with COVID-19: Retrospective cohort study. The BMJ 2021; 372: n693

13. Saygili ES, Karakiliç E, Mert E, Șener A, Mirci A. Preadmission usage of metformin and mortality in COVID-19 patients including the postdischarge period. Irish Journal of Medical Science Ir J Med Sci. 2021 Oct 29. doi: 10.1007/s11845-021-02823-9 [Epub ahead of print].

14. Khwaja A. KDIGO Clinical Practice Guidelines for Acute Kidney Injury. Nephron Clin Pract 2012; 120: c179-84.

15. Levey AS, Stevens LA, Schmid CH, Zhang YL, Castro AF, Feldman HI, et al. A new equation to estimate glomerular filtration rate. Ann Intern Med 2009; 150: 604-12.

16. Brieghel C, Ellekvist P, Lund ML, Søborg C, Walsted ES, Thomsen JJ, et al. Prognostic factors of 90-day mortality in patients hospitalised with COVID-19. Dan Med J 2021; 68: A09200705.

17. Carrasco-Sánchez FJ, López-Carmona MD, Martínez-Marcos FJ, PérezBelmonte LM, Hidalgo-Jiménez A, Buonaiuto V, et al. Admission hyperglycaemia as a predictor of mortality in patients hospitalized with COVID-19 regardless of diabetes status: data from the Spanish SEMI-COVID-19 Registry. Ann Med 2021; 53: 103-16.

18. Lima-Martínez MM, Carrera Boada C, Madera-Silva MD, Marín W, Contreras M. COVID-19 and diabetes: A bidirectional relationship. Clin Investig Arterioscler 2021; 33: 151-7.

19. Clement S, Braithwaite SS, Magee MF, Ahmann A, Smith EP, Schafer RG, et al. Management of diabetes and hyperglycemia in hospitals. Diabetes Care 2004; 27: 553-91.

20. Dhatariya K, Corsino L, Umpierrez GE. Management of Diabetes and Hyperglycemia in Hospitalized Patients. Endotext. South Dartmouth (MA): MDText.com, Inc; 2020. Available at: http://www.ncbi.nlm.nih. gov/pubmed/25905318

21. Akirov A, Grossman A, Shochat T, Shimon I. Hyperglycemia on admission and hospitalization outcomes in patients with atrial fibrillation. Clinical Cardiol 2017; 40: 1123-8.
22. Vogelzang M, Nijboer JMM, van der Horst ICC, Zijlstra F, ten Duis HJ, Nijsten MWN. Hyperglycemia Has a Stronger Relation with Outcome in Trauma Patients than in Other Critically III Patients. J Trauma 2006; 60: 873-9.

23. Sung J, Bochicchio GV, Joshi M, Bochicchio K, Tracy K, Scalea TM. Admission hyperglycemia is predictive if outcome in critically ill trauma patients. J Trauma 2005; 59: 80-3.

24. Bornstein SR, Rubino F, Khunti K, Mingrone G, Hopkins D, Birkenfeld $\mathrm{AL}$, et al. Practical recommendations for the management of diabetes in patients with COVID-19. Lancet Diabetes Endocrinol 2020; 8: 546-50.

25. Björk M, Melin EO, Frisk T, Thunander M. Admission glucose level was associated with increased short-term mortality and length-of-stay irrespective of diagnosis, treating medical specialty or concomitant laboratory values. Eur J Intern Med 2020; 75: 71-8.

26. Dirkali A, van der Ploeg T, Nangrahary M, Cornel JH, Umans VAWM. The impact of admission plasma glucose on long-term mortality after STEMI and NSTEMI myocardial infarction. Int J Cardiol 2007; 121: 215-7.

27. Itzhaki Ben Zadok O, Kornowski R, Goldenberg I, Klempfner R, Toledano Y, Biton Y, et al. Admission blood glucose and 10-year mortality among patients with or without pre-existing diabetes mellitus hospitalized with heart failure. Cardiovascular Diabetol 2017; 16: 1-9.

28. Stentz FB, Umpierrez GE, Cuervo R, Kitabchi AE. Proinflammatory cytokines, markers of cardiovascular risks, oxidative stress, and lipid peroxidation in patients with hyperglycemic crises. Diabetes 2004; 53: 2079-86.

29. Boden G. Gluconeogenesis and glycogenolysis in health and diabetes. J Investig Med 2004; 52: 375-8.

30. Siegelaar SE, Holleman F, Hoekstra JBL, DeVries JH. Glucose variability; does it matter? Endocr Rev 2010; 31: 171-82.

31. Rao S, Lau A, So HC. Exploring diseases/traits and blood proteins causally related to expression of ACE2, the putative receptor of SARSCoV-2: A mendelian randomization analysis highlights tentative relevance of diabetes-related traits. Diabetes Care 2020; 43: 1416-26.

32. Gallo Marin B, Aghagoli G, Lavine K, Yang L, Siff EJ, Chiang SS, et al. Predictors of COVID-19 severity: A literature review. Rev Med Virol 2021; 31: 1-10.

33. Moghissi ES, Korytkowski MT, DiNardo M, Einhorn D, Hellman R, Hirsch IB, et al. AACE/ADA Consensus Statement. Diabetes Care 2009; 15: 353-69.

34. Moghissi ES, Korytkowski MT, DiNardo M, Einhorn D, Hellman R, Hirsch IB, et al. American Association of Clinical Endocrinologists and American Diabetes Association Consensus Statement on Inpatient Glycemic Control. Diabetes Care 2009; 32: 1119-31. 